statement that condoms encourage pre-marital sex, more than 80 percent agreed that condom prevents spread of HIV infection. Personal contact with PLHIV does not seem to have positive influence with willingness to advocate condom use.

Conclusion Church leaders agreed that they have responsibilities towards HIV prevention but were restricted by the Church doctrine. Disobedience to Biblical teachings was believed to result in HIV infection which is seen as something sinful. Misconceptions around HIV persist irrespective of awareness programs within the Church. Sex education from early childhood, using Church media and educating Church leaders were recommended for effective HIV prevention.

\section{P5.18 A GLOBAL ESTIMATE OF THE ACCEPTABILITY OF PRE- EXPOSURE PROPHYLAXIS FOR HIV AMONG MEN WHO HAVE SEX WITH MEN: A SYSTEMATIC REVIEW AND META-ANALYSIS}

'Lei Zhang, ${ }^{2}$ Peng Peng. ${ }^{1}$ Melbourne Sexual Health Centre, Australia; ${ }^{2}$ School of Public Health, Monash University, Melbourne, Australia

\subsection{6/sextrans-2017-053264.634}

Introduction Pre-exposure prophylaxis (PrEP) is a new biomedical intervention for HIV prevention. This study systematically reviews the acceptability of PrEP among men who have sex with men (MSM) worldwide.

Methods We searched PubMed database to identify Englishlanguage articles published between July 2007 and July 2016, which reported the acceptability of PrEP and associated population characteristics. Meta-analysis was conducted to estimate a pooled rate of acceptability, and meta-regression and subgroup analysis were used to analyse heterogeneities.

Results Sixty-eight articles were included. The estimated acceptance of PrEP was 58.7\% (95\% confidence internal (CI): $53.4 \%-63.8 \%$ ) among MSM worldwide and showed no significant difference between developed and developing countries. MSM who were younger ( $4 / 5$ studies, range of adjusted odds ratio $(\mathrm{aOR})=1.49-3.47)$, better educated $(7 / 7$ studies, $\mathrm{aOR}=1.49-7.70)$, wealthier (3/3 studies, $\mathrm{aOR}=1.31-13.03)$, previously aware of $\operatorname{PrEP}(4 / 4$ studies, aOR $=1.33-3.30)$ and had greater self-perceived risk of HIV infection (4/5 studies, $\mathrm{aOR}=1.20-4.67)$ showed significantly higher acceptance of PrEP. Male sex workers (84.0\% [26.3-98.7\%]\%] were more likely to accept PrEP than general MSM. Self-perceived low efficacy and concern about side effects, adherence, affordability, and stigma from health providers and society were main barriers for accepting PrEP.

Conclusion This review identifies a moderate acceptability of PrEP in MSM both developed and developing country settings. Efficacy, individuals' perception of HIV risk and level of experienced stigma determine its acceptance.

\section{P5.19 HEALTH NEEDS OF WOMEN WHO HAVE SEX WITH WOMEN AND ACCESS TO HEALTH SERVICES}

Marli Teresinha Cassamassimo Duarte, Ana Paula Freneda de Freitas, Juliane Andrade, Mariana Alice De Oliveira Ignacio. Universidade Estadual Paulista, Brazil

\subsection{6/sextrans-2017-053264.635}

Introduction Access and host are essential in order to satisfactorily intervene in the population health and the Brazilian literature has few studies approaching the access to services and sexual and reproductive care among women who have sex with women (WSW). The aim of this study was to analyse the access to health services and sexual and reproductive health care of WSW in public health policies.

Methods Cross-sectional, descriptive and analytical study. The data were obtained by interview, gynaecological and blood laboratory examination for sexually transmitted infections (STI) diagnosis, from January 2015 to December 2016.The descriptive statistics and the quantitative method of Bardin were used to analyse the access to health services. Sexual and reproductive health care was analysed by linear and multiple regression models.

Results The intentional sample was 149 WSW and the median age was 27 years-old (18-62). There was a predominance of white women (74.5\%), non-union (73.2\%), who had paid activity (73.2\%) and eight years or more of completed study $(96,0 \%)$. The majority $(84.0 \%)$ used the public health service, $22.6 \%$ sought preventive actions. The difficulties encountered in the health services care were reported by 77 women, resulted in 118 responses with $28.8 \%$ associated with difficulty by long waiting time and $7.6 \%$ by lack of preparation of the professional/service for MSM.Regarding sexual and reproductive care, $71.8 \%$ had sexual intercourse after consumption of alcohol and/or illicit drugs, $12.1 \%$ did not use a condom and $51.7 \%$ had at least one STI. Age was a factor associated with the care score $(p=0.001, \beta=1.36 \mathrm{CI}=0.57$ 2.15) and no differences were observed in the MSM health care score with a history of sexual intercourse with men compared to those who only had women.

Conclusion Beside the difficulties of access to health services already experienced by women in general, there is a high programmatic in addition to individual and social vulnerability. This study indicates the need for effective strategies for the proposed policies for WSW.

\section{P5.20 HPV VACCINATION INTENTION AMONG FEMALE SEX WORKERS IN AMSTERDAM, THE NETHERLANDS}

${ }^{1}$ MF Schim Van Der Loeff, E Marra, ${ }^{1} \mathrm{~L}$ Van Dam, ${ }^{1} \mathrm{~N}$ Kroone, ${ }^{1} \mathrm{M}$ Craanen, ${ }^{1} \mathrm{~A}$ Van Dijk, ${ }^{1} \mathrm{CJ}$ Alberts, ${ }^{2} \mathrm{GD}$ Zimet, ${ }^{3} \mathrm{TGWM}$ Paulussen, ${ }^{1} \mathrm{~T}$ Heijman, ${ }^{1} \mathrm{~A}$ Hogewoning, ${ }^{1} \mathrm{GJB}$ Sonder, ${ }^{1} \mathrm{HJC}$ De Vries. ${ }^{1}$ GGD Amsterdam, The Neederlands; Indiana University, USA; ${ }^{2} T N O$

\subsection{6/sextrans-2017-053264.636}

Introduction Female sex workers (FSW) are at risk for HPVinduced diseases but are currently not targeted by the HPV vaccination program in the Netherlands. We explored the determinants of their intention to get vaccinated against HPV in case vaccination would be offered to them in the near future.

Methods In 2016, FSW aged >18 years having an STI consultation with the Prostitution and Health Centre (P and G292) in Amsterdam, either at the clinic or at their working location, were invited to complete a questionnaire assessing the socio-psychological determinants of their HPV vaccination intention (scale ranging from -3 to +3 ). Determinants of HPV vaccination intention were assessed with uni- and multivariable linear regression. Additionally, we explored the effect of out-of-pocket payment on intention.

Results Between May and September 2016, 293 FSW participated; 98 (34\%) worked in clubs/private houses/massage salons, $111(38 \%)$ worked at 'prostitution windows', and 81 $(28 \%)$ worked as escorts or from home. The median age was 
29 years (IQR 25-37). HPV vaccination intention was relatively high (mean 2.0; 95\% CI:1.8-2.2). In multivariable analysis attitude $(\beta=0.6 ; 95 \% \mathrm{CI}: 0.5-0.7)$, descriptive norm $(\beta=0.3 ; 95 \%$ CI:0.2-0.4), self-efficacy $(\beta=0.2 ; 95 \%$ CI:0.1$0.3)$, beliefs $(\beta=0.1 ; 95 \% \mathrm{CI}: 0.0-0.2)$ and anticipated regret $(\beta=0.1 ; 95 \%$ CI:0.0-0.2) were the strongest predictors of HPV vaccination intention. Demographic variables did not improve the multivariable regression model. The explained variance in the model (R2) was 0.54 . HPV vaccination intention decreased significantly when vaccination would require out-of-pocket payment (€50 mean: 1.2 (95\%CI: 0.8-1.7); $€ 100$ mean: 1.6 (95\%CI: 1.1-2.0); $€ 200$ mean: $1.0(95 \%$ CI: $0.5-1.5$ ); $€ 350$ mean: 0.2 (95\%CI: $-0.2-0.7$ ).

Conclusion HPV vaccination intention among FSW in Amsterdam appears to be very high. The included socio-psychological factors explained most of the variance in HPV vaccination intention among FSW. Out-of-pocket payment had a significant negative effect on HPV vaccination intention.

\section{P5.21 FISHERMEN AS A SUITABLE POPULATION FOR HIV INTERVENTION TRIALS}

${ }^{1}$ Njeri Mbugua, ${ }^{2}$ Elizabeth Ann Bukusi. 'Kenya Medical Research Institute/Nairobi University/ Kenyatta Hospital/Kenya Women with HIVIAIDS; ${ }^{2}$ Kenya Medical Research Institute, Nairobi University, Kenya

\subsection{6/sextrans-2017-053264.637}

Introduction Suitable populations to sustain continued evaluation of HIV and sexually transmitted infection (STI) prevention interventions are required. We sought to determine whether fishermen are a suitable population for HIV intervention trials.

Methods In a cross-sectional descriptive survey, we selected 250 fishermen from proportional to size sampled boats. We collected socioeconomic and behavioural information, and specimens for HIV, herpes simplex virus (HSV-2), syphilis, gonorrhoea, chlamydia and human papillomavirus (HPV) tests from consenting participants.

Results One third of the fishermen had concurrent sexual partnerships and two thirds were involved in transactional sex. About $70 \%$ were involved in extramarital sex with only one quarter using condoms in their three most recent sexual encounters. HIV prevalence was $26 \%$ and HSV-2 and HPV was $57 \%$. Over $98 \%$ were willing to participate in a future HIV prevention clinical trial.

Conclusion Fishermen are a high-risk group for HIV/STI infections that may be suitable for HIV prevention trials. A cohort study would be useful to measure the incidence of HIV/STIs to ultimately determine the feasibility of enrolling this population in an HIV/STI prevention clinical trial.

\section{P5.22 THE IMPORTANCE OF HAVING AN ADOLESCENT HIV UNIT CASE REPORT AT JOS UNIVERSITY TEACHING HOSPITAL (JUTH), JOS PLATEAU STATE, NIGERIA}

Ogudiegwu Catherine Chinyere. Jos University Teaching Hospital, Nigeria

\subsection{6/sextrans-2017-053264.638}

Issue JUTH has one of the largest HIV treatment centres in North central Nigeria currently has 529 children enrolled into the program,498 on treatment. Since inception in 2004,92 children had virologic failure of their treatment and 45 transferred to the Adult ART program.I present the case of one of our children that have grown into Adulthood who defaulted her treatment,failed treatment regimen, got pregnant from HIV negative sex partner and had her baby is free of HIV. Body: The child was enrolled into the program at age of 10 and she lost mother to HIV when she was 9 years old. Her 1 st line drug regimen was Zidovudine, Niverapine and lamivudine which she was not adherent to.At age 17, she admitted that she had a sexual partner who was later invited counselled and tested, his HIV antibody test was negative.She was later transferred to the adult clinic at the age of 18 years with viral load of 52568 copies/ml while CD4 count of 345 cells $/ \mathrm{mm}$. She got pregnant also immediately from another partner but failed to access Prevention of child transmission of HIV (PMTCT)for fear of being scourned by the health workers. She had a vaginal delivery at 39 weeks.

Intervention Baby was enrolled at the paediatric unit,had Nevirapine prophylaxis for 6 weeks and was breastfeed baby exclusively for 6 months.Condoms use was inconsistent and she was also on daily contraceptive. Her baby recorded HIV negative results for DNA PCR (Deoxyribonucleic acid polymerase chain reaction) results at week 6,12, and 24 and Post breast feeding DNA PCR test.Baby is still incare for followup. Sex patner was counselled,tested for HIV, antibody testing result was HIV negative, and he was subsequently enrolled for the prevention program(PrEP).

Lessons learnt There is need to establish an Adolecent ART clinic where reproductive health can be discussed freely and healthworkers should encourage instead of being judgmental and also we need to educate them on HIV infection and reinfection,drug adherence and also encourage abstinence.

\section{P5.23 HOW MUCH CAN HIV TRANSMISSION BE REDUCED IN HIGH-RISK MSM BY TARGETING TESTING TO DETECT AND TREAT PRIMARY HIV INFECTION (PHI)? ANALYSIS OF A COHORT STUDY USING AN INDIVIDUAL-BASED MODEL}

${ }^{1}$ Peter White, ${ }^{2} J u l i e$ Fox, ${ }^{1} J o n a t h a n$ Weber, ${ }^{1}$ Helen Ward, ${ }^{1}$ Sarah Fidler. ${ }^{1}$ Imperial College London, UK; ${ }^{2}$ Guys and St Thomas' NHS Trust/Kings College London, UK

\subsection{6/sextrans-2017-053264.639}

Introduction HIV incidence remains high in UK MSM, and cost-effective combination prevention is needed. We estimated numbers of infections averted by targeting testing for highlyinfectious primary HIV infection (PHI) in high-risk MSM.

Methods A cohort study of 98 MSM (participation rate 94\%; zero loss to follow-up) recently infected with HIV recorded sexual behaviour in the 3 months pre- and post-HIV diagnosis. A stochastic individual-based model calculated numbers of HIV-transmission events expected to occur with and without the effects of (i) immediate ART in those who chose to receive it and (ii) behaviour changes reported post-diagnosis by those who did and did not receive ART. The model incorporates different types of sex-act, condom use, and distinguishes between regular and casual partners.

Results If PHI lasts for 3 months, testing is monthly, and viral load is suppressed by ART after 3 months with an initial rapid decline in the first 2 weeks, then from the 73 patients who took ART the reduction in transmission would be $\sim 75 \%$, from $22-33$ to $5-8$ events, and in the remaining 25 patients 